\section{A Randomized, Open-Label Comparison of Once-Weekly Insulin Icodec Titration Strategies Versus Once-Daily Insulin Glargine U100}

Diabetes Care 2021;44:1-9 | https://doi.org/10.2337/dc20-2878

\section{OBJECTIVE}

Insulin icodec is a novel once-weekly basal insulin analog. This trial investigated the efficacy and safety of icodec using different once-weekly titration algorithms.

\section{RESEARCH DESIGN AND METHODS}

This was a phase 2, randomized, open-label, 16-week, treat-to-target study. Insulin-naïve adults $(n=205)$ with type 2 diabetes and $\mathrm{HbA}_{1 \mathrm{c}} \mathbf{7 - 1 0 \%}$ while treated with oral glucose-lowering medications initiated once-weekly icodec titrations $A$ (prebreakfast self-measured blood glucose target $80-130 \mathrm{mg} / \mathrm{dL}$; adjustment \pm 21 units/week; $n=51)$, В (80-130 mg/dL; \pm 28 units/week; $n=51)$, or C (70-108 $\mathrm{mg} / \mathrm{dL} ; \pm 28$ units/week; $\boldsymbol{n}=\mathbf{5 2}$ ), or once-daily insulin glargine U100 (IGlar U100) $(80-130 \mathrm{mg} / \mathrm{dL} ; \pm 4$ units/day; $n=51)$, all titrated weekly. Percentage of time in range (TIR) (70-180 mg/dL) during weeks 15 and 16 was measured using continuous glucose monitoring.

\section{RESULTS}

TIR improved from baseline (means: A, 57.0\%; B, 55.2\%; C, 51.0\%; IGlar U100, 55.3\%) to weeks 15 and 16 (estimated mean: A, 76.6\%; B, 83.0\%; C, 80.9\%; IGlar U100, 75.9\%). TIR was greater for titration B than for IGlar U100 (estimated treatment difference $7.08 \%$-points; $95 \% \mathrm{Cl} 2.12$ to $12.04 \% ; P=0.005)$. No unexpected safety signals were observed. Level 2 hypoglycemia ( $<54 \mathrm{mg} / \mathrm{dL}$ ) was low in all groups $(0.05$, $0.15,0.38,0.00$ events per patient-year of exposure for icodec titrations $A, B$, and C and IGlar U100, respectively), with no episodes of severe hypoglycemia.

\section{CONCLUSIONS}

Once-weekly icodec was efficacious and well tolerated across all three titration algorithms investigated. The results for icodec titration A $(80-130 \mathrm{mg} / \mathrm{dL} ; \mathbf{2 1}$ units/week) displayed the best balance between glycemic control and risk of hypoglycemia.

Treatment adherence remains a challenge in patients with type 2 diabetes, and the issue is particularly pronounced in those prescribed insulin (1). Barriers to insulin therapy initiation and adherence include both the reluctance of health care providers to prescribe insulin and the resistance among patients in taking insulin (2).
Ildiko Lingvay, ${ }^{1,2}$ John B. Buse, ${ }^{3}$ Edward Franek, ${ }^{4}$ Melissa V. Hansen, ${ }^{5}$ Mette M. Koefoed, ${ }^{5}$ Chantal Mathieu, ${ }^{6}$ Jeremy Pettus, ${ }^{7}$ Karolina Stachlewska, ${ }^{5}$ and Julio Rosenstock ${ }^{8}$
${ }^{1}$ Endocrinology Division, Department of Internal Medicine, University of Texas Southwestern Medical Center, Dallas, TX

${ }^{2}$ Department of Population and Data Sciences, University of Texas Southwestern Medical Center, Dallas, $T X$

${ }^{3}$ School of Medicine, University of North Carolina at Chapel Hill, Chapel Hill, NC

${ }^{4}$ Mossakowski Medical Research Centre, Warsaw, Poland

${ }^{5}$ Novo Nordisk A/S, Søborg, Denmark

${ }^{6}$ Clinical and Experimental Endocrinology, University of Leuven, Leuven, Belgium

${ }^{7}$ School of Medicine, University of California, San Diego, San Diego, CA

${ }^{8}$ Dallas Diabetes Research Center at Medical City, Dallas, $T X$

Corresponding author: Ildiko Lingvay, ildiko. lingvay@utsouthwestern.edu

Received 25 November 2020 and accepted 15 February 2021

Clinical trial reg. no. NCT03951805, clinicaltrials. gov

This article contains supplementary material online at https://doi.org/10.2337/figshare.14054921.

(c) 2021 by the American Diabetes Association. Readers may use this article as long as the work is properly cited, the use is educational and not for profit, and the work is not altered. More information is available at https://www. diabetesjournals.org/content/license.

See accompanying articles, $p p$. XXX and XXX. 
Hesitance and fear concerning injections and the additional complexity of timing of insulin administration contribute to these barriers (3-5). In addition, the efficacy and safety of insulin treatments are highly dependent on, among other things, optimal titration, which is often not followed in clinical practice because the resources and support systems are not available to the same extent as in randomized clinical trials. Indeed, insulin dose, timing, and glycemic targets have a greater impact on efficacy and safety profiles of insulin than differences among insulin formulations (6). Reducing the frequency of treatment administration may decrease both provider and patient reluctance to insulin initiation, increase patient adherence and quality of life, and decrease treatment burden, ultimately leading to improved glycemic control. Treatment with an injectable once-weekly glucagon-like peptide 1 receptor agonist has been associated with a significant improvement in glycemic control, treatment adherence, treatment satisfaction, and quality of life compared with once-daily therapies (7-11), and presumably a similar effect may be expected for a once-weekly basal insulin in clinical practice.

Insulin icodec is a novel once-weekly basal insulin analog that strongly but reversibly binds to albumin. The major mode of protraction is due to the formation of an essentially inactive albumin-bound depot, which slowly releases active insulin icodec over time (12). Pharmacokinetic and pharmacodynamic data show that insulin icodec has a halflife of $\sim 1$ week with a glucose-lowering effect close to evenly distributed within a 1-week dosing interval (13). Guidance on titration algorithms is needed for the efficacious and safe use of this novel insulin by clinicians and patients. In a recently published 26-week phase 2 study in insulin-naïve patients with type 2 diabetes, insulin icodec displayed glucoselowering effects and safety profile comparable to those of once-daily insulin glargine U100 (IGlar U100) (14).

To better inform titration and dosing strategies for this novel once-weekly insulin, we examined three different insulin icodec titration algorithms in comparison with once-daily IGlar U100 in insulin-naïve patients with type 2 diabetes. Glucose-lowering effects were investigated primarily with continuous glucose monitoring (CGM), an assessment method that is rapidly growing in popularity in clinical practice but novel and rarely used to measure a primary end point in clinical trials.

\section{RESEARCH DESIGN AND METHODS}

\section{Research Design}

This was a randomized, active-controlled, parallel-group, multicenter, multi-national, open-label, phase 2, treatto-target trial conducted in seven countries (Croatia, Germany, Hungary, Poland, Slovakia, Spain, and the U.S.). The trial consisted of a 2-week screening period, 16 weeks of treatment, and a 5week follow-up.

The trial was conducted in accordance with current applicable international and national regulations and ethics requirements and was performed in accordance with the Declaration of Helsinki, applicable recommendation from the International Council for Harmonisation of Technical Requirements for Pharmaceuticals for Human Use's guidelines for good clinical practice, and the International Organization for Standardization ISO 14155 . The protocol, consent form, and other relevant documents were approved by the appropriate independent review boards or independent ethics committees.

\section{Patients}

Patients were eligible if they were adults aged 18-75 years who received a diagnosis of type 2 diabetes at least 180 days prior to screening, were treated with metformin with or without dipeptidyl peptidase 4 inhibitor (DPP4i) and/ or sodium-glucose cotransporter 2 inhibitors (SGLT2i), and had glycated hemoglobin $\left(\mathrm{HbA}_{1 \mathrm{c}}\right)$ of 7.0-10.0\% (53.0$85.8 \mathrm{mmol} / \mathrm{mol})$. All inclusion and exclusion criteria are detailed in Supplementary Table 1 . Written informed consent was obtained for all participants.

\section{Randomization}

Patients were randomized 1:1:1:1 to receive subcutaneous injections of onceweekly insulin icodec following one of three titration algorithms (icodec titrations $\mathrm{A}, \mathrm{B}$, or $\mathrm{C}$, outlined below) or oncedaily IGlar U100 (Supplementary Fig. 1A). Randomization was performed centrally with an interactive Web response system, and patients were stratified based on SGLT2i treatment. The full analysis set (FAS) consisted of all randomized patients. The safety analysis set (SAS) consisted of all patients exposed to at least one dose of trial product.

\section{Procedures}

Insulin icodec (Novo Nordisk, Bagsværd, Denmark) was initiated at 70 units weekly and IGlar U100 (Sanofi, Paris, France) at 10 units daily, equivalent to 70 units per week. Prebreakfast selfmeasured blood glucose (SMBG) target values used for titration were based on the American Diabetes Association (ADA)-recommended preprandial plasma glucose target of $4.4-7.2 \mathrm{mmol} / \mathrm{L}$ (80-130 mg/dL) (15) or a tighter target of $3.9-6.0 \mathrm{mmol} / \mathrm{L}(70-108 \mathrm{mg} / \mathrm{dL})$ that was used in prior treat-to-target studies including a previously published 26week phase 2 trial $(14,16)$. SMBG was assessed with a blood glucose meter as plasma-equivalent values of capillary whole blood glucose. The titration algorithms were as follows and were based on three prebreakfast SMBG values on the 2 days leading up to titration and on the day of titration: IGlar U100, prebreakfast SMBG target $4.4-7.2 \mathrm{mmol} / \mathrm{L}$ (80-130 mg/dL), adjustment \pm 4 units/ day; insulin icodec titration $A$, prebreakfast SMBG target $4.4-7.2 \mathrm{mmol} / \mathrm{L}$ (80-130 mg/dL), adjustment \pm 21 units/ week (same SMBG target as for IGlar U100 and smaller dose increments); insulin icodec titration $B$, prebreakfast SMBG target 4.4-7.2 $\mathrm{mmol} / \mathrm{L}$ (80-130 $\mathrm{mg} / \mathrm{dL}$ ), adjustment \pm 28 units/week (same SMBG target and equivalent dose increments as for IGlar U100); and insulin icodec titration $C$, prebreakfast SMBG target $3.9-6.0 \mathrm{mmol} / \mathrm{L}$ (70-108 mg/dL), adjusted \pm 28 units/ week (tighter and lower SMBG target than for IGlar U100 and equivalent dose increments).

Insulin doses were adjusted weekly (Supplementary Fig. 1B). If any of the three prebreakfast SMBG values were below the lower limit of the target range, titration was based on the lowest recorded value. If all three SMBG values were above the lower limit of the target range, titration was based on the mean of the three measurements. Both insulin icodec (700 units $/ \mathrm{mL}$ ) and IGlar U100 (100 units $/ \mathrm{mL}$ ) were administered 


\begin{tabular}{|c|c|c|c|c|c|}
\hline & $\begin{array}{c}\text { Insulin icodec } \\
\text { titration A } \\
(n=51)\end{array}$ & $\begin{array}{c}\text { Insulin icodec } \\
\text { titration B } \\
(n=51)\end{array}$ & $\begin{array}{c}\text { Insulin icodec } \\
\text { titration C } \\
(n=52)\end{array}$ & $\begin{array}{c}\text { IGlar U100 } \\
(n=51)\end{array}$ & $\begin{array}{c}\text { Total } \\
(N=205)\end{array}$ \\
\hline Age, years & $59.8(9.1)$ & $61.2(8.0)$ & $61.4(8.0)$ & $60.2(8.1)$ & $60.7(8.3)$ \\
\hline Male, \% & 52.9 & 54.9 & 53.8 & 52.9 & 53.7 \\
\hline Duration of type 2 diabetes, years & $9.8(7.2)$ & $9.6(4.9)$ & $9.2(4.4)$ & $11.8(6.8)$ & $10.1(6.0)$ \\
\hline Body weight, kg & $91.4(17.6)$ & $90.4(18.0)$ & $87.3(14.0)$ & $86.4(17.1)$ & $88.9(16.7)$ \\
\hline $\mathrm{BMI}, \mathrm{kg} / \mathrm{m}^{2}$ & $32.3(4.8)$ & $31.4(4.7)$ & $30.8(3.8)$ & $30.6(4.7)$ & $31.3(4.5)$ \\
\hline TIR, \% & $57.0(28.0)$ & $55.2(26.3)$ & $51.0(27.4)$ & $55.3(29.2)$ & $54.6(27.6)$ \\
\hline $\mathrm{FPG}, \mathrm{mg} / \mathrm{dL}$ & $174(33)$ & $180(38)$ & $177(41)$ & $168(42)$ & 175 (39) \\
\hline $\mathrm{HbA}_{1 \mathrm{c}}, \%$ & $8.0(0.7)$ & $8.1(0.8)$ & $8.2(0.9)$ & $8.2(0.8)$ & $8.1(0.8)$ \\
\hline $\mathrm{HbA}_{1 \mathrm{c}}, \mathrm{mmol} / \mathrm{mol}$ & $63.9(7.8)$ & $64.9(8.4)$ & $65.6(10.3)$ & $65.7(9.1)$ & $65.0(8.9)$ \\
\hline \multicolumn{6}{|l|}{ Oral antidiabetic drug, $n(\%)^{*}$} \\
\hline Metformin monotherapy & $20(39.2)$ & 16 (31.4) & $25(48.1)$ & $17(33.3)$ & $78(38.0)$ \\
\hline Metformin + SGLT2i & $12(23.5)$ & $11(21.6)$ & $11(21.2)$ & 10 (19.6) & $44(21.5)$ \\
\hline Metformin + DPP4i & $14(27.5)$ & $19(37.3)$ & $9(17.3)$ & $16(31.4)$ & $58(28.3)$ \\
\hline Metformin + SGLT2i + DPP4i & $5(9.8)$ & $5(9.8)$ & $7(13.5)$ & $8(15.7)$ & $25(12.2)$ \\
\hline
\end{tabular}

Data are means (SD) unless otherwise stated. Insulin icodec titration A: titration of insulin icodec to a prebreakfast SMBG target of 4.4-7.2 $\mathrm{mmol} / \mathrm{L}(80-130 \mathrm{mg} / \mathrm{dL})$ with dose adjustment of \pm 21 units. Insulin icodec titration B: titration of insulin icodec to a prebreakfast SMBG target of $4.4-7.2 \mathrm{mmol} / \mathrm{L}(80-130 \mathrm{mg} / \mathrm{dL})$ with dose adjustment of \pm 28 units. Insulin icodec titration C: titration of insulin icodec to a prebreakfast SMBG target of $3.9-6.0 \mathrm{mmol} / \mathrm{L}(70-108 \mathrm{mg} / \mathrm{dL}$ ) with dose adjustment of \pm 28 units. IGlar U100: titration of IGlar U100 to a prebreakfast SMBG target of $4.4-7.2 \mathrm{mmol} / \mathrm{L}(80-130 \mathrm{mg} / \mathrm{dL})$ with dose adjustment of \pm 4 units. *At screening.

subcutaneously in the thigh with the 3-mL FlexTouch and the 3-mL SoloStar prefilled pen injectors, respectively. It was recommended that study drugs be injected at any time of the day but at

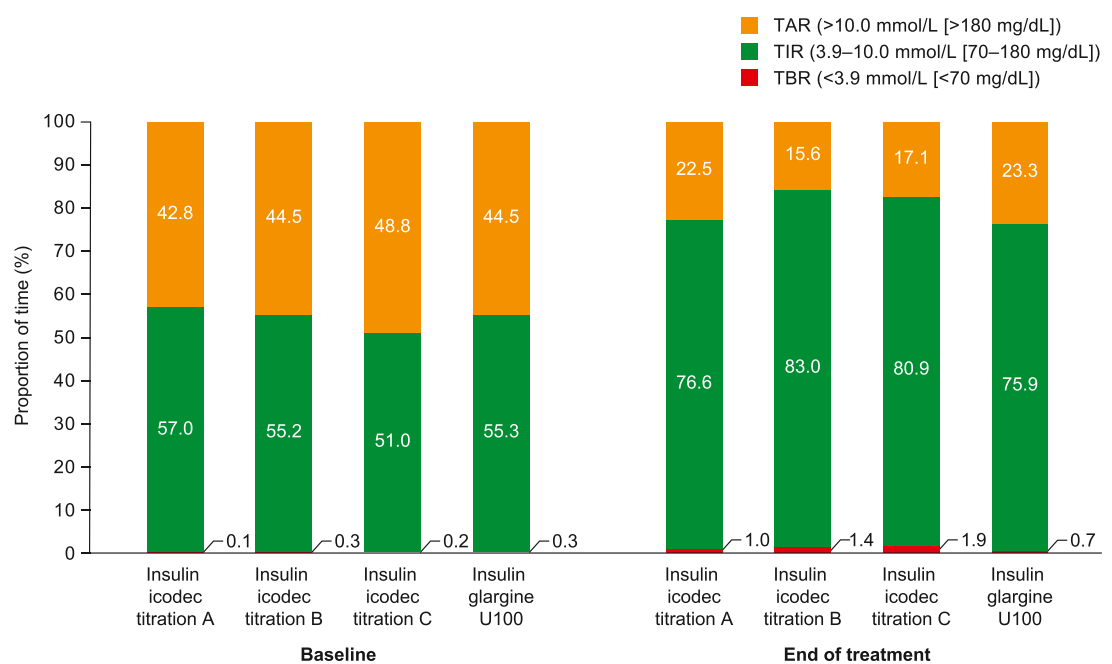

Figure 1-TIR 3.9-10.0 mmol/L (70-180 mg/dL) at baseline and during the last 2 weeks of treatment (weeks 15 and 16) (FAS, $N=205$ ). Baseline values are observed mean values. End-of-treatment values represent estimated mean values. The numbers of patients who had missing or $<70 \%$ CGM measurements during the last 2 weeks of treatment (weeks 15 and 16) were two for titration $A$, one for titration $B$, two for titration $C$, and one for IGlar U100. Insulin icodec titration A: titration of insulin icodec to a prebreakfast SMBG target of 4.4-7.2 mmol/L (80-130 $\mathrm{mg} / \mathrm{dL}$ ) with dose adjustment of \pm 21 units. Insulin icodec titration $B$ : titration of insulin icodec to a prebreakfast SMBG target of $4.4-7.2 \mathrm{mmol} / \mathrm{L}(80-130 \mathrm{mg} / \mathrm{dL})$ with dose adjustment of \pm 28 units. Insulin icodec titration $\mathrm{C}$ : titration of insulin icodec to a prebreakfast SMBG target of 3.9-6.0 mmol/L (70-108 mg/dL) with dose adjustment of \pm 28 units. IGlar U100: titration of IGlar U100 to a prebreakfast SMBG target of $4.4-7.2 \mathrm{mmol} / \mathrm{L}(80-130 \mathrm{mg} / \mathrm{dL})$ with dose adjustment of \pm 4 units. TAR, time above range; TBR, time below range. screening to the end-of-treatment visit. CGM data were uploaded at each site visit. Both patients and investigators were blinded to CGM data throughout the study and these data were not used for insulin dose titration or reporting of hypoglycemic episodes. Patients performed SMBG assessment once a day (prebreakfast) to inform dose titration decisions and to mitigate hypoglycemic risk. In addition, patients were required to measure SMBG if they suspected having low blood glucose. It was required that at least $70 \%$ of the planned CGM measurements, during the last 2 weeks of treatment, be available for end point data to be included in the analysis.

The primary end point was percentage of time in range (TIR) (3.9-10.0 $\mathrm{mmol} / \mathrm{L}$ [70-180 $\mathrm{mg} / \mathrm{dL}]$ ) during the last 2 weeks of treatment (weeks 15 and 16), measured by CGM (17). Supportive secondary end points were as follows: changes from baseline to week 16 in $\mathrm{HbA}_{1 \mathrm{c}}$, fasting plasma glucose (FPG), and body weight; weekly insulin dose during the last 2 weeks of treatment (weeks 15 and 16); and number of on-treatment adverse events (AEs) from baseline to week 21 and number of self-reported hypoglycemic episodes documented by SMBG or assessed as the requirement of 
external assistance for recovery. Hypoglycemic episodes were classified as "alert" values (level 1: blood glucose $<3.9 \mathrm{mmol} / \mathrm{L}[<70 \mathrm{mg} / \mathrm{dL}]$ and $\geq 3.0$ $\mathrm{mmol} / \mathrm{L}[\geq 54 \mathrm{mg} / \mathrm{dL}]$ ), combined clinically significant (level 2: blood glucose $<3.0 \mathrm{mmol} / \mathrm{L}[<54 \mathrm{mg} / \mathrm{dL}]$ ), and severe hypoglycemia (level 3: severe cognitive impairment requiring external assistance) or severe hypoglycemia only (level 3). AEs of interest included major adverse cardiovascular events, hypersensitivity reactions, and deaths; these were reviewed by a blinded, independent adjudication committee.

\section{Statistical Analysis}

To achieve a width of the $95 \% \mathrm{Cl}$ for TIR 3.9-10.0 mmol/L (70-180 mg/dL) of $2.5 \mathrm{~h}$ (corresponding to a TIR of $\sim 10 \%$ ) for a 24-h period for any pairwise comparison between any insulin icodec treatment group and IGlar U100 with a probability of $80 \%$ and an assumed SD of $3.0 \mathrm{~h}$ (corresponding to a TIR of $\sim 12.5 \%$ ), it was required that at least 200 patients be randomized to reach the target sample size of 50 patients per treatment group.

TIR for each individual was calculated as the number of recorded measurements in the range $3.9-10.0 \mathrm{mmol} / \mathrm{L}$ (70-180 $\mathrm{mg} / \mathrm{dL}$ ) divided by the total number of recorded measurements over 14 days, multiplied by 100 .

The primary estimand, which was the "trial product estimand" (18), was defined as the mean difference in the primary end point measures between each of the three insulin icodec titrations and IGlar U100 if all patients had adhered to randomized treatment without initiation of rescue medication and had $70 \%$ of the planned CGM measurements recorded. A more detailed explanation of the rationale for estimand and of the estimand used in this trial is provided in the Supplementary Material. For TIR, the response during the last 2 weeks of treatment (weeks 15 and 16) was analyzed with an ANCOVA model, with treatment and SGLT2i use as fixed factors and baseline TIR value as a covariate. Missing end point values were imputed with use of multiple imputation based on own treatment group, with baseline TIR as a covariate. Each imputed data set was analyzed separately, and estimates were combined with use of Rubin's rules (19).
Supportive secondary efficacy end points were analyzed in the same way as the primary end point, with the exception of the mean weekly insulin dose during the last 2 weeks of treatment, which was log transformed and analyzed with an ANOVA model, without baseline value as covariate. Missing data for secondary end points were imputed based on own treatment group with a sequential conditional regression approach, including all postbaseline values whenever intermediate assessments of the end point were available. Intermittent missing values were imputed with the Markov chain Monte Carlo method. Because this was a phase 2 trial and exploratory in nature, no adjustments were made for multiplicity.

The on-treatment period was defined as the period from the date of first study drug dose until the last follow-up visit or the last dosing day of randomized treatment plus 5 weeks (for IGlar U100) or 6 weeks (for insulin icodec)whichever came first-and represents the time period in which patients were considered exposed to trial product.

Data were analyzed with SAS software, version 9.4 (SAS Institute Inc., Cary, NC). No data monitoring committees oversaw the study. This trial was registered on ClinicalTrials.gov (clinical trial reg. no. NCT03951805).

\section{Data and Resource Availability}

The data sets generated during or analyzed during the current trial are available from the corresponding author on reasonable request.

\section{RESULTS}

Between May 9 and August 9, 2019, 259 patients were screened, of whom 205 were randomized to one of the treatment groups (icodec titration $\mathrm{A}$, $n=51$; icodec titration $\mathrm{B}, n=51$; icodec titration C, $n=52$; IGlar U100, $n=51$ ) and received at least one dose of study drug (FAS, $N=205$; SAS, $N=$ 205) (Supplementary Fig. 2). Only one patient in the icodec titration $A$ group withdrew from the trial, and one patient initiated rescue medication in the icodec titration $\mathrm{C}$ group. The numbers of patients who had missing or $<70 \%$ CGM measurements during the last 2 weeks of treatment (weeks 15 and 16) were two for icodec titration $A$, one for icodec titration B, two for icodec titration C, and one for IGlar U100. Demographics and baseline characteristics were generally similar across treatment groups (Table 1).

The mean percentage TIRs (3.9-10.0 $\mathrm{mmol} / \mathrm{L}$ [70-180 $\mathrm{mg} / \mathrm{dL}]$ ) at baseline for icodec titrations A, B, C, and IGlar U100 were $57.0 \%, 55.2 \%, 51.0 \%$, and $55.3 \%$, respectively, and all increased at weeks 15 and 16 to estimated means of $76.6 \%, 83.0 \%, 80.9 \%$, and $75.9 \%$, respectively (Fig. 1). In comparison with IGlar U100, TIR for icodec titration B was longer, with an estimated treatment difference (ETD) of 7.08\%-points (95\% Cl 2.12 to $12.04 \% ; P=0.005$ ), corresponding approximately to an extra 102 min spent in target glycemic range daily. In comparison with IGlar U100, TIR was numerically greater for icodec titration C (ETD 5.01\%-points; $95 \% \mathrm{Cl}-0.04$ to $10.05 \% ; P=0.05)$ and similar for icodec titration A (ETD $0.76 \%$-points; $95 \% \mathrm{Cl}-4.28$ to $5.80 \%$; $P=0.77)$. Data on coefficient of variation are presented in Supplementary Table 2, and time below and time above range are presented in Fig. 1.

End-of-treatment (week 16) results for key secondary end points are presented in Supplementary Table 3. The estimated mean changes from baseline to week 16 in $\mathrm{HbA}_{1 \mathrm{c}}$ for icodec titrations $\mathrm{A}, \mathrm{B}, \mathrm{C}$, and IGlar U100 were -1.0\%-point (-10.9 $\mathrm{mmol} / \mathrm{mol}),-1.2 \%$-points $(-13.4 \mathrm{mmol} /$ $\mathrm{mol}),-1.4 \%$-points $(-15.1 \mathrm{mmol} / \mathrm{mol})$, and $-1.0 \%$-point $(-11.1 \mathrm{mmol} / \mathrm{mol})$, respectively (Fig. 2A). The ETD for $\mathrm{HbA}_{1 \mathrm{c}}$ was $0.02 \%$-points (95\% Cl -0.20 to $0.24 \%$ ) for titration A versus IGlar U100, $-0.20 \%$ points $(95 \% \mathrm{Cl}-0.42$ to $0.02 \%$ ) for titration $\mathrm{B}$ versus IGlar U100, and $-0.36 \%$-points (95\% Cl -0.58 to $-0.14 \%$ ) for titration C versus IGlar U100 (Supplementary Table 3). At week 16, the proportion of patients who achieved $\mathrm{HbA}_{1 \mathrm{c}}<7.0 \%$ (53.0 mmol/ mol) was $46.0 \%$ for icodec titration $A$, $64.0 \%$ for icodec titration B, $70.6 \%$ for icodec titration C, and $51.0 \%$ for IGlar U100 (Supplementary Fig. 3).

The estimated mean change from baseline to week 16 in FPG was -2.2 $\mathrm{mmol} / \mathrm{L}(-40 \mathrm{mg} / \mathrm{dL})$ for icodec titration A, $-2.4 \mathrm{mmol} / \mathrm{L}(-44 \mathrm{mg} / \mathrm{dL})$ for icodec titration $\mathrm{B},-3.0 \mathrm{mmol} / \mathrm{L}(-54 \mathrm{mg} / \mathrm{dL})$ for icodec titration $\mathrm{C}$, and $-2.3 \mathrm{mmol} / \mathrm{L}$ (-42 mg/dL) for IGlar U100 (Supplementary Fig. 4). 

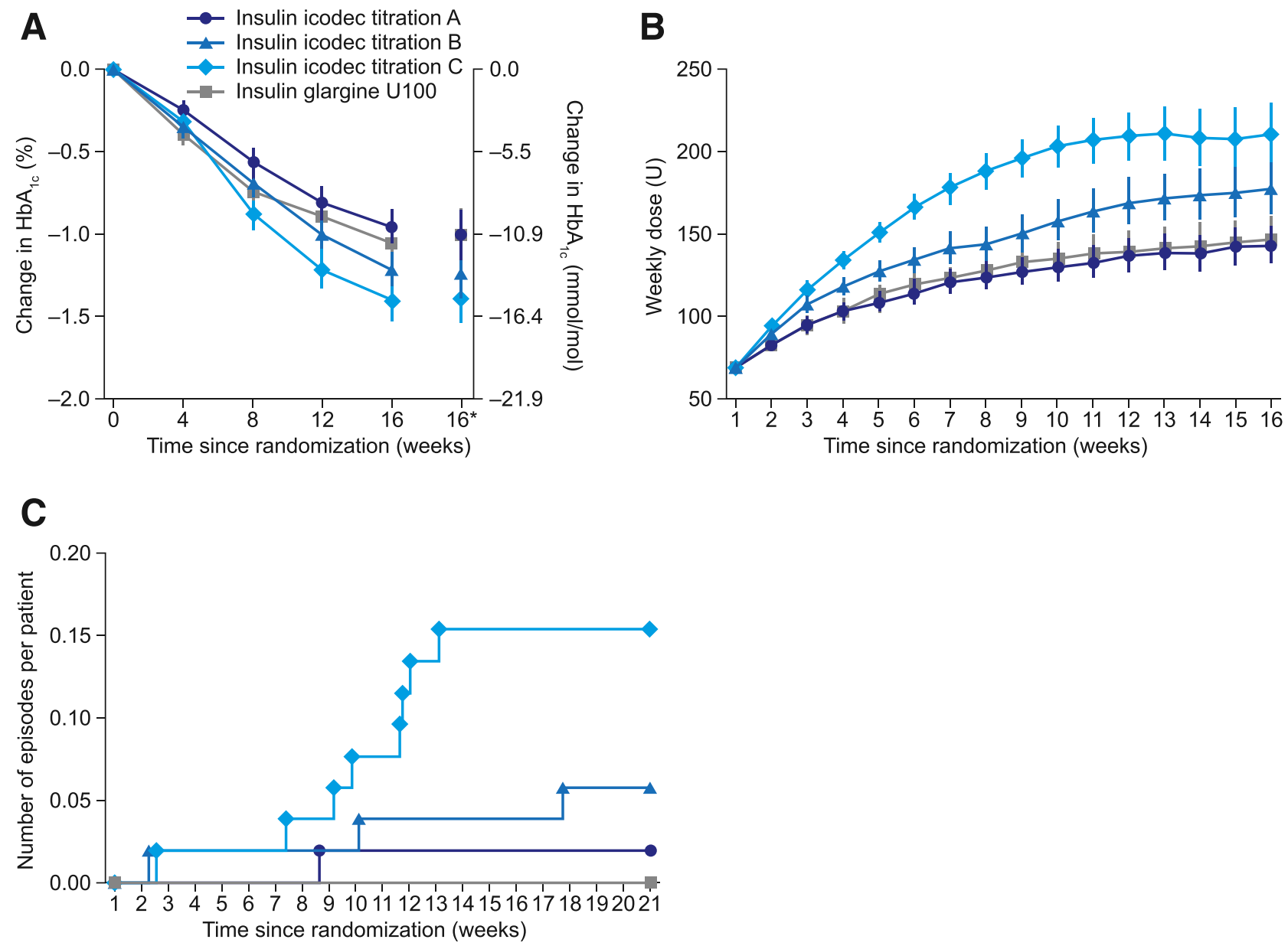

Figure 2-Changes in key parameters during the 16-weeks study (FAS, $N=205)$. Mean change in $\mathrm{HbA}_{1 \mathrm{c}}$ from baseline to week $16(A)$, mean weekly insulin dose over time $(B)$, and mean cumulative function of number of severe (level 3 ) and clinically significant (level 2) hypoglycemic events when subjects were on treatment $(C)$. Observed data: mean (symbol) \pm SEM $(A)$ and geometric mean (symbol) \pm SEM on log-scale backtransformed (B). Insulin icodec titration A: titration of insulin icodec to a prebreakfast SMBG target of 4.4-7.2 mmol/L (80-130 mg/dL) with dose adjustment of \pm 21 units. Insulin icodec titration B: titration of insulin icodec to a prebreakfast SMBG target of $4.4-7.2 \mathrm{mmol} / \mathrm{L}$ ( $80-130 \mathrm{mg} / \mathrm{dL}$ ) with dose adjustment of \pm 28 units. Insulin icodec titration C: titration of insulin icodec to a prebreakfast SMBG target of 3.9-6.0 mmol/L (70-108 mg/dL) with dose adjustment of \pm 28 units. IGlar U100: titration of IGlar U100 to a prebreakfast SMBG target of $4.4-7.2 \mathrm{mmol} / \mathrm{L}$ (80-130 mg/dL) with dose adjustment of \pm 4 units. $U$, units. ${ }^{*}$ Estimated mean values and the corresponding $\mathrm{Cls}$ at week 16 derived based on multiple imputation.

The estimated mean weekly doses during weeks 15 and 16 were 142.5 units, 176.4 units, 208.9 units, and 145.6 units for icodec titrations A, B, C, and IGlar U100, respectively (Fig. 2B). There was an estimated increase of 0.9 , 1.1 , and $1.3 \mathrm{~kg}$ body wt over 16 weeks for icodec titrations $A, B$, and $C$, respectively, and of $0.6 \mathrm{~kg}$ for IGlar U100 (Supplementary Table 3).

Mean fasting SMBG, used as the basis for titration but not measured as a study end point, decreased in all treatment groups below the upper limits of the respective titration target ranges over the course of the study. The largest change was observed for icodec titration C (prebreakfast SMBG target 3.9-6.0 $\mathrm{mmol} / \mathrm{L} \quad[70-108 \mathrm{mg} / \mathrm{dL}])$
(Supplementary Fig. 5), reflecting the study design.

The incidences and rates of hypoglycemic episodes are presented in Table 2. The rates of on-treatment level 1 hypoglycemic episodes per patient year of exposure from baseline through follow-up (week 21) were low across treatment groups. Rates of combined clinically significant (level 2) and severe (level 3) hypoglycemic episodes were low for all insulin icodec titrations (0.05, 0.15 , and 0.38 events per patient-year of exposure for icodec titrations $A, B$, and $C$, respectively) with no clustering of episodes over the on-treatment period (Fig. 2C); none occurred in the IGlar U100 group. No severe (level 3) hypoglycemic episodes occurred in any treatment group (Table 2). During weeks 15 and 16, the estimated mean daily time spent below range $(<3.9$ $\mathrm{mmol} / \mathrm{L}$ ) was $1.0 \%$ for titration $\mathrm{A}, 1.4 \%$ for titration $B, 1.9 \%$ for titration $C$, and $0.7 \%$ for IGlar U100, corresponding to $\sim 14,20,27$, and 10 min per day, respectively (Fig. 1). There was no apparent clustering of level 1 hypoglycemic events in the week following the day of injection for icodec titrations $A$ and $B$. Clustering of level 2 hypoglycemia in the week following the day of injections could not be assessed because there were too few events. Similar results were observed for the period from baseline to week 16 (data not shown).

The incidences of on-treatment AEs were similar between all four treatment 


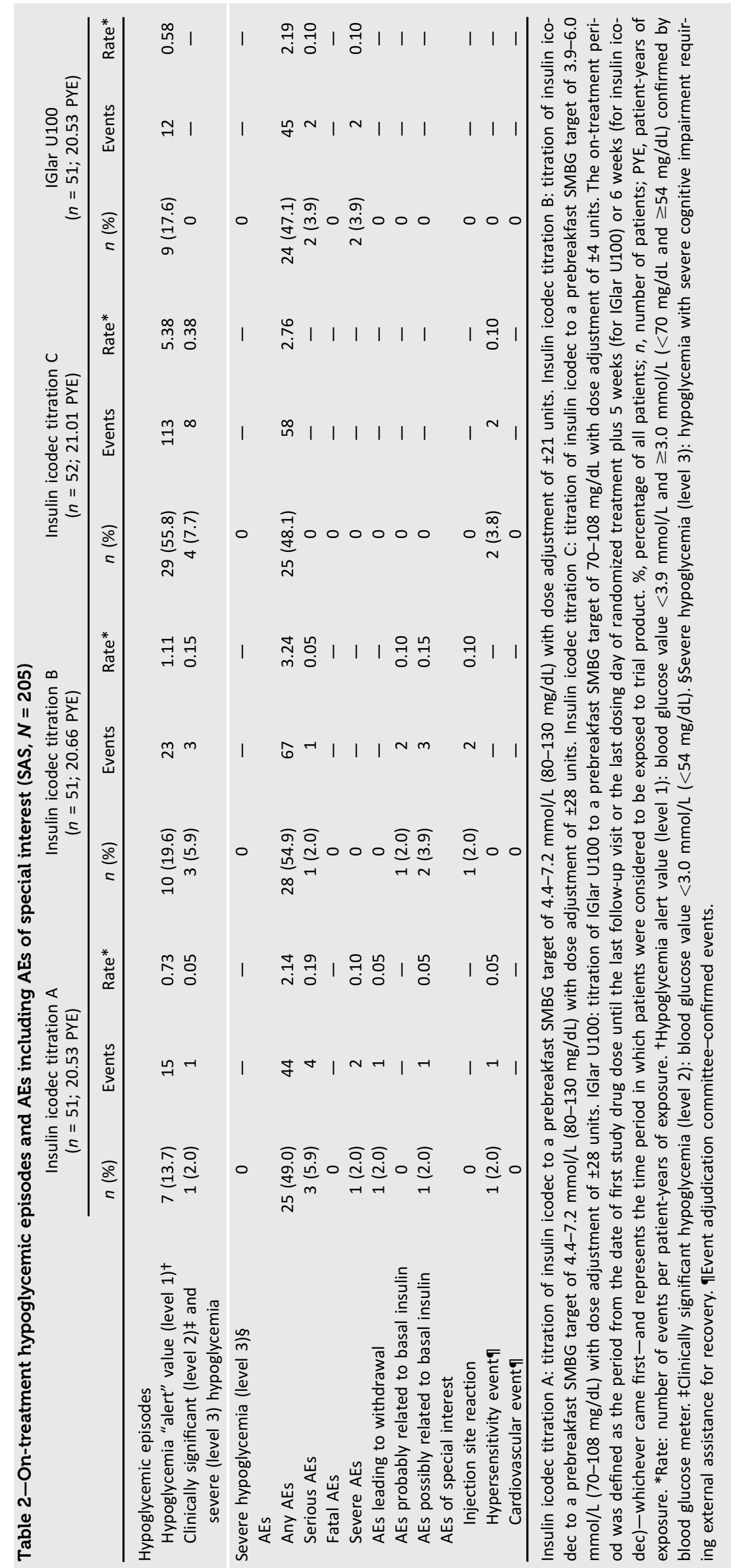


groups (Table 2). Across treatments, most AEs were considered unlikely to be related to the trial product and were nonserious and of mild or moderate severity. Serious AEs were reported in three patients receiving icodec titration $A$, one patient receiving icodec titration $B$, no patients receiving icodec titration $C$, and two patients receiving IGlar U100. All serious AEs were considered unlikely to be related to study drug.

Overall, eight hypersensitivity events were reported in five patients (icodec titration A, two events; icodec titration $B$, four events; icodec titration $C$, two events) and three events were confirmed following adjudication (icodec titration A, one event; icodec titration C, two events); all were localized, not at the injection site, and not considered to be related to study drug. Injection site reactions were reported in one patient (icodec titration B, two events, both mild). No cardiovascular events were reported, no $A E$ clusters were observed, and no new safety signals were identified.

\section{CONCLUSIONS}

Insulin icodec is a novel once-weekly basal insulin analog with the potential to increase treatment adherence by improving convenience and quality of life of patients with diabetes by reducing the number of injections from 365 to 52 per year. However, there are new aspects inherent to the use of a once-weekly basal insulin analog to consider, such as optimizing titration. A preprandial plasma glucose target of $4.4-7.2 \mathrm{mmol} / \mathrm{L}$ (80-130 $\mathrm{mg} / \mathrm{dL})$ was investigated because it reflects real-life practice and ADA guidance. Indeed, lower and tighter targets tested in randomized clinical trials are seldom used in clinical practice because primary care practitioners and specialists might have concerns regarding hypoglycemia when using such strict targets. Icodec titration B was intended to provide a direct comparator to IGlar U100, with the same plasma glucose target and corresponding dose increments, while icodec titration A was included for investigation of the effect of smaller dose increments. The more stringent target used in icodec titration $C$ was included not only to explore the effect of such a stringent target but also to serve as a reference for comparison of the present data with those from the 26-week phase 2 trial (14).

In this phase 2 treat-to-target study, we found that once-weekly insulin icodec effectively lowered glucose levels, similarly to what was seen with oncedaily IGlar U100. Subtle differences were noted across the three icodec titration algorithms evaluated.

In comparison of IGlar U100 and insulin icodec titrated to the same target with use of the same titration increments (titration B), a greater TIR was achieved with insulin icodec, corresponding approximately to an extra 102 min spent in the target glycemic range daily. $\mathrm{HbA}_{1 \mathrm{c}}$ was lower with insulin ico$\mathrm{dec}$, and there was a slightly higher rate of level 2 and level 1 hypoglycemia, albeit the hypoglycemia event rate was very low overall. Titration to attain a more stringent glucose target of 3.9-6.0 $\mathrm{mmol} / \mathrm{L}(70-108 \mathrm{mg} / \mathrm{dL})$, as used in titration algorithm $\mathrm{C}$, was also associated with a higher rate of hypoglycemia in comparison with IGlar U100 titrated to a target of $4.4-7.2 \mathrm{mmol} / \mathrm{L}$ (80-130 mg/ $\mathrm{dL}$ ), while TIR was numerically greater and $\mathrm{HbA}_{1 \mathrm{c}}$ lower with insulin icodec. Lastly, titration algorithm $A$, which used the same glucose target of 4.4-7.2 $\mathrm{mmol} / \mathrm{L}(80-130 \mathrm{mg} / \mathrm{dL})$ and a smaller titration increment of \pm 21 units in comparison with IGlar U100, which was titrated in \pm 4 unit increments (weekly equivalent \pm 28 units), led to comparable glucose control, as assessed by both TIR and $\mathrm{HbA}_{1 \mathrm{c}}$, and comparable hypoglycemia rates. Interestingly, the final daily insulin dose in the titration algorithm B was numerically larger, by $\sim 21 \%$, compared with that in the IGlar U100 group despite the identical titration parameters used in the two groups. This finding contrasts with that in another phase 2 trial that compared insulin icodec with IGlar U100 and used a double-blind design with 26 weeks of follow-up. In that study, the average daily dose of insulin used during the last 2 weeks of treatment was $\sim 19 \%$ lower in the icodec group in comparison with IGlar U100 while the same weekly titration parameters were used in both groups (prebreakfast SMBG target 70-108 mg/dL; adjustment \pm 14 or 28 units weekly equivalent) (14). Thus, it is prudent to postpone any conclusions regarding dose equivalency between these products until the results of larger ongoing phase 3 studies are available. Interestingly, for icodec titration A, which used a smaller titration increment than titration $B$, there was a weekly dose comparable with that of IGlar U100 and as a likely consequence, icodec titration A showed a profile similar to that of IGlar U100 with respect to TIR, $\mathrm{HbA}_{1 \mathrm{c}}, \mathrm{FPG}$, and SMBG. Therefore, it is likely that ultimately the daily insulin dose achieved is the primary driver of glucose-lowering efficacy and hypoglycemia risk-rather than a specific titration algorithm.

A modest increase in weight was observed in all groups and was in line with expectations when insulin treatment was initiated in an insulin-naïve population. The magnitude of weight gain appeared to be insulin dose dependent, yet the differences between groups were small.

Insulin icodec was well tolerated in this study, with similar incidence of AEs reported in all treatment groups. The rates of hypoglycemia were generally low compared with other treat-to-target trials $(16,20,21)$. It was reassuring that no severe (level 3) hypoglycemic events were reported for any treatment group throughout the entire study duration and that the time spent below range ( $<3.9 \mathrm{mmol} / \mathrm{L}$ ) during the last 2 weeks of treatment (weeks 15 and 16) was below the $4 \%$ target recommended by the International Consensus on Time in Range across treatment groups (17).

In summary, titration algorithm A appears to provide the most optimal balance between glycemic control and hypoglycemia across the three titration algorithms tested. These results were very informative for determination of the titration algorithm used in the ongoing phase 3 trials (clinical trial reg. no. NCT04460885, NCT04795531, and NCT04760626, ClinicalTrials.gov).

The strengths of this study include the following: its randomized, multicenter design; the very low treatment discontinuation rate; and the assessment of glycemic control via TIR with the novel use of CGM in a relatively large clinical trial in patients with type 2 diabetes. While $\mathrm{HbA}_{1 \mathrm{c}}$ is still considered the gold standard in assessment of glycemic control, CGM data are increasingly used in clinical practice and rapidly replacing $\mathrm{HbA}_{1 \mathrm{c}}$ as a more comprehensive tool to assess glycemic control, as they provide a more detailed and granular assessment of various factors that play into glycemic control beyond an average 
glucose level. Of these, increased TIR has now been associated with relative risk reductions for various complications of diabetes, including diabetic retinopathy, peripheral neuropathy, cardiovascular autonomic neuropathy, and allcause and CVD-related morality (22-27). Previous publications have indicated that a $5 \%$ increase in TIR was associated with an estimated $0.3-0.4 \%$ decrease in $\mathrm{HbA}_{1 \mathrm{c}}(17,28-30)$; yet, TIR is becoming more clinically relevant. To our knowledge, this is one of the largest studies in patients with type 2 diabetes to use CGM with TIR as a primary end point. In addition, improved glycemic control was achieved in all four treatment groups despite the short duration of the study. The short duration of 16 weeks, which may not be long enough to see the effect of a stable maintenance phase, is an acknowledged limitation of this study, and longer studies will follow in the phase 3 program. However, a plateau was observed in all treatment groups from week 14 for SMBG, suggesting that stability was achieved when TIR was evaluated during weeks 15 and 16. In addition, the study population might not be representative of the typical patient population initiating insulin, among whom there is often a delay in insulin initiation and hereby often a higher $\mathrm{HbA}_{1 \mathrm{c}}$ than what was noted at baseline for the current study population. Finally, it should be noted that, because for icodec titration $C$ there was a lower, more stringent titration target, 3.9-6.0 $\mathrm{mmol} / \mathrm{L}(70-108 \mathrm{mg} / \mathrm{dL})$, than for the other treatment groups, direct comparisons between icodec titration $C$ and IGlar U100 should be cautiously interpreted in the appropriate context.

In conclusion, this study suggests that insulin icodec, a novel, once-weekly basal insulin analog, was efficacious and well tolerated across all three titration algorithms. The titration algorithm with the ADA-recommended preprandial plasma glucose target and the smallest dose increments appears to represent the best balance between glycemic control and risk of hypoglycemia and the profile most comparable with IGlar U100 with regards to TIR, $\mathrm{HbA}_{1 \mathrm{c}}, \mathrm{FPG}$, and SMBG. Insulin icodec has the potential to provide patients and medical teams with a more convenient alternative to once-daily basal insulin and thus decrease the treatment burden in patients with type 2 diabetes requiring treatment with basal insulin.

Acknowledgments. The authors are grateful to the participants of this study, to Kamilla Begtrup of Novo Nordisk $A / S$, Søborg, Denmark, for her input on the study concept and design, and to Dr. Jim Purvis and Dr. Nicolas Bertheleme of Oxford PharmaGenesis, Oxford, U.K. (supported by Novo Nordisk A/S), for writing assistance.

Funding. J.B.B.'s effort in this study was supported in part through grants from the NIH (UL1TR002489, P30DK124723). J.B.B. is supported by grants from the National Institutes of Health, Patient-Centered Outcomes Research Institute (PCORI), and ADA. The medical writing agency that assisted the authors with writing the manuscript was funded by Novo Nordisk A/S.

Duality of Interest. Novo Nordisk funded the trial and was responsible for trial design and data analysis. I.L. received research funding, advisory/consulting fees, or other support from: Novo Nordisk, Eli Lilly, Sanofi, AstraZeneca, Boehringer Ingelheim, Janssen, Intercept, Intarcia, Target RWE, Merck, Pfizer, Novartis, GI Dynamics, Mylan, Mannkind, Valeritas, Bayer, and Zealand Pharma. J.B.B.'s contracted consulting fees and travel support for contracted activities are paid to the University of North Carolina by Adocia, AstraZeneca, Dance Biopharm, Eli Lilly, Fortress Biotech, MannKind, NovaTarg, Novo Nordisk, Sanofi, Senseonics, vTv Therapeutics, and Zafgen in addition to grant support from NovaTarg, Novo Nordisk, Sanofi, Tolerion and VTV Therapeutics. J.B.B. is also a consultant to Cirius Therapeutics, CSL Behring, Fortress Biotech, Mellitus Health, Neurimmune AG, Pendulum Therapeutics, Stability Health, and Zealand Pharma. He holds stock/options in Mellitus Health, Pendulum Therapeutics, PhaseBio, and Stability Health. E.F. received advisory/consulting fees from AstraZeneca, Bioton, Boehringer Ingelheim, Novartis, and Novo Nordisk and lecture fees from AstraZeneca, Bioton, Boehringer Ingelheim, Eli Lilly, Novartis, Novo Nordisk, and Polfa Tarchomin. C.M. serves or has served on the advisory panel for Novo Nordisk, Sanofi, Merck Sharp \& Dohme, Eli Lilly, Novartis, AstraZeneca, Boehringer Ingelheim, Hanmi Pharmaceuticals, Roche, Medtronic, ActoBio Therapeutics, Pfizer, and UCB. Financial compensation for these activities has been received by University of Leuven, and University of Leuven has received research support for her from Medtronic, Novo Nordisk, Sanofi, Merck Sharp and Dohme, Eli Lilly, Roche, Abbott, ActoBio Therapeutics, and Novartis. She serves or has served on the speakers' bureau for Novo Nordisk, Sanofi, Merck Sharp \& Dohme, Eli Lilly, Boehringer Ingelheim, AstraZeneca, and Novartis. Financial compensation for these activities has been received by University of Leuven. J.P. has received consulting fees from Sanofi, Novo Nordisk, Diasome, Mannkind, and Lexicon. J.R. has participated in advisory panels for Boehringer Ingelheim Pharmaceuticals, Intarcia Therapeutics, Applied Therapeutics, Janssen Pharmaceuticals, Eli Lilly, Novo Nordisk, Sanofi, and Oramed. He has received research support from
GlaxoSmithKline, Janssen Pharmaceuticals, Pfizer, Intarcia Therapeutics, Genentech, Merck, Eli Lilly, Novartis, Novo Nordisk, Sanofi, and Oramed. M.V.H., M.M.K., and K.S. are employees of Novo Nordisk and hold share options and/or shares. No other potential conflicts of interest relevant to this article were reported.

Author Contributions. M.M.K. participated in the study concept and design. All authors were involved in the acquisition, analysis, and interpretation of data and participated in preparing the manuscript with the support of medical writing services. All authors had full access to all the data, read and approved the submitted version of the manuscript, and had final responsibility for the decision to submit the manuscript for publication. I.L. is the guarantor of this work and, as such, had full access to all the data in the study and takes responsibility for the integrity of the data and the accuracy of the data analysis.

Prior Presentation. Parts of this study were presented in abstract form at the 54th Annual Meeting of the European Association for the Study of Diabetes, 21-25 September 2020.

\section{References}

1. Perez-Nieves M, Boye KS, Kiljanski J, Cao D, Lage MJ. Adherence to basal insulin therapy among people with type 2 diabetes: a retrospective cohort study of costs and patient outcomes. Diabetes Ther 2018;9:1099-1111

2. Peyrot $M$, Rubin RR, Lauritzen $T$, et al.; International DAWN Advisory Panel. Resistance to insulin therapy among patients and providers: results of the cross-national Diabetes Attitudes, Wishes, and Needs (DAWN) study. Diabetes Care 2005;28:2673-2679

3. Polonsky WH, Henry RR. Poor medication adherence in type 2 diabetes: recognizing the scope of the problem and its key contributors. Patient Prefer Adherence 2016;10:1299-1307

4. Sarbacker GB, Urteaga EM. Adherence to insulin therapy. Diabetes Spectr 2016;29:166-170

5. Yehl K. AADE practice paper in brief: diabetes educators play a critical role in successful insulin management. AADE Pract 2018;6:36-37

6. Davies MJ, D'Alessio DA, Fradkin J, et al. Management of hyperglycemia in type 2 diabetes, 2018. A consensus report by the American Diabetes Association (ADA) and the European Association for the Study of Diabetes (EASD). Diabetes Care 2018;41:2669-2701

7. Mody R, Huang $Q, Y u M$, et al. Adherence, persistence, glycaemic control and costs among patients with type 2 diabetes initiating dulaglutide compared with liraglutide or exenatide once weekly at 12-month follow-up in a real-world setting in the United States. Diabetes Obes Metab 2019;21:920-929

8. Morieri $M L$, Rigato $M$, Frison $V$, et al. Effectiveness of dulaglutide vs liraglutide and exenatide once-weekly. A real-world study and meta-analysis of observational studies. Metabolism 2020;106:154190

9. Polonsky WH, Fisher L, Hessler D, Bruhn D, Best $\mathrm{JH}$. Patient perspectives on once-weekly medications for diabetes. Diabetes Obes Metab 2011;13:144-149

10. Nguyen $H$, Dufour $R$, Caldwell-Tarr A. Glucagon-like peptide-1 receptor agonist (GLP1RA) therapy adherence for patients with type 2 
diabetes in a Medicare population. Adv Ther 2017;34:658-673

11. Best JH, Boye KS, Rubin RR, Cao D, Kim TH, Peyrot M. Improved treatment satisfaction and weight-related quality of life with exenatide once weekly or twice daily. Diabet Med 2009;26:722728

12. Nishimura E, Kjeeldsen T, Hubalek F, et al. Molecular and biological properties of insulin icodec, a new insulin analog designed to give a long half-life suitable for once-weekly dosing (Abstract). Diabetes 2020;69(Suppl. 1):AXXX

13. Hövelmann $U$, Brøndsted L, Kristensen NR, et al. Insulin icodec: an insulin analog suited for once-weekly dosing in type 2 diabetes (Abstract). Diabetes 2020;69(Suppl. 1):AXXX

14. Rosenstock J, Bajaj HS, Janež A, et al.; NN1436-4383 Investigators. Once-weekly insulin for type 2 diabetes without previous insulin treatment. N Engl J Med 2020;383:2107-2116

15. American Diabetes Association. 6. Glycemic targets: Standards of Medical Care in Diabetes2018. Diabetes Care 2018;41(Suppl. 1):S55-S64

16. Rosenstock J, Davies M, Home PD, Larsen J, Koenen C, Schernthaner G. A randomised, 52week, treat-to-target trial comparing insulin detemir with insulin glargine when administered as add-on to glucose-lowering drugs in insulinnaive people with type 2 diabetes. Diabetologia 2008;51:408-416

17. Battelino T, Danne T, Bergenstal RM, et al. Clinical targets for continuous glucose monitoring data interpretation: recommendations from the international consensus on time in range. Diabetes Care 2019;42:1593-1603

18. Aroda VR, Saugstrup T, Buse JB, Donsmark M, Zacho J, Davies MJ. Incorporating and interpreting regulatory guidance on estimands in diabetes clinical trials: the PIONEER 1 randomized clinical trial as an example. Diabetes Obes Metab 2019; 21:2203-2210

19. Little RJA, Rubin DB. Statistical Analysis With Missing Data. New York, John Wiley \& Sons, 1987 20. Swinnen SG, Dain MP, Aronson R, et al. A 24week, randomized, treat-to-target trial comparing initiation of insulin glargine once-daily with insulin detemir twice-daily in patients with type 2 diabetes inadequately controlled on oral glucoselowering drugs. Diabetes Care 2010;33:11761178

21. Ratner RE, Gough SCL, Mathieu C, et al. Hypoglycaemia risk with insulin degludec compared with insulin glargine in type 2 and type 1 diabetes: a pre-planned meta-analysis of phase 3 trials. Diabetes Obes Metab 2013;15:175-184

22. Lu J, Ma X, Shen $Y$, et al. Time in range is associated with carotid intima-media thickness in type 2 diabetes. Diabetes Technol Ther 2020;22: 72-78

23. Lu J, Ma X, Zhou J, et al. Association of time in range, as assessed by continuous glucose monitoring, with diabetic retinopathy in type 2 diabetes. Diabetes Care 2018;41:2370-2376
24. Mayeda L, Katz R, Ahmad I, et al. Glucose time in range and peripheral neuropathy in type 2 diabetes mellitus and chronic kidney disease. BMJ Open Diabetes Res Care 2020;8:e000991

25. Guo Q, Zang $P, X u S$, et al. Time in range, as a novel metric of glycemic control, is reversely associated with presence of diabetic cardiovascular autonomic neuropathy independent of $\mathrm{HbA} 1 \mathrm{c}$ in Chinese type 2 diabetes. J Diabetes Res 2020;2020:5817074

26. Lu J, Wang $C$, Shen $Y$, et al. Time in range in relation to all-cause and cardiovascular mortality in patients with type 2 diabetes: a prospective cohort study. Diabetes Care 2021;44: 549-555

27. Ranjan AG, Rosenlund SV, Hansen TW Rossing P, Andersen S, Nørgaard K. Improved time in range over 1 year is associated with reduced albuminuria in individuals with sensor-augmented insulin pump-treated type 1 diabetes. Diabetes Care 2020;43:2882-2885

28. Beck RW, Bergenstal RM, Cheng P, et al. The relationships between time in range, hyperglycemia metrics, and HbA1c. J Diabetes Sci Technol 2019;13:614-626

29. Vigersky RA, McMahon C. The relationship of hemoglobin $\mathrm{A} 1 \mathrm{C}$ to time-in-range in patients with diabetes. Diabetes Technol Ther 2019;21:81-85

30. Beck RW, Bergenstal RM, Riddlesworth TD, et al. Validation of time in range as an outcome measure for diabetes clinical trials. Diabetes Care 2019;42:400-405 